\title{
Choques transitorios y de largo plazo en el producto interno bruto y precios en el departamento del Meta ${ }^{1}$
}

\section{Transitory and long-term shocks in the gross domestic product and prices in the Meta department}

DOI: http://dx.doi.org/10.17981/econcuc.39.1.2018.06

Artículo de investigación. Fecha de recepción: 03/11/2017 Fecha de aceptación: 25/05/2018

\author{
Astrid León-Camargo (iD \\ Universidad de los Llanos (Colombia) \\ aleonc@unillanos.edu.co
}

Para citar este artículo:

León-Camargo, A. (2018). Choques transitorios y de largo plazo en el producto interno bruto y precios en el departamento del Meta. Económicas CUC, 39(1). 87-104. DOI: http://dx.doi.org/10.17981/econcuc.39.1.2018.06

\section{Resumen}

Este artículo presenta el impacto de corto y largo plazo en el Producto Interno Bruto (PIB) y los precios en el departamento del Meta en Colombia entre 1990 y 2015. Se utiliza la metodología de los vectores autorregresivos estructurales (VAR) con datos del DANE. Los resultados muestran que los choques de oferta tienen un impacto positivo de largo plazo sobre el PIB del departamento del Meta e influyen de forma negativa sobre los precios, mientras los choques de demanda solo tienen efecto sobre los precios y no influyen sobre el PIB. La única crisis con impacto significativo es la crisis financiera sobre los precios.

Palabras claves: VAR estructural, ciclo económico, choques transitorios, choques de largo plazo, descomposición de Blanchard y Quah.

\section{Abstract}

This article presents the impact shortlong on GDP and prices in the department of Meta in Colombia, between 1990's and 2015 's. The methodology of the structural auto-regressive vectors (VAR) is used, with data from DANE. The results show that supply shocks have a long-term positive impact on the GDP of the Meta department and negatively influence prices, while demand's shocks only have an effect on prices and do not influence GDP. The only crisis with a significant impact is the financial crisis on prices.

Keywords: Structural VAR, economic cycle, transient shocks, long term collisions, Blanchard and Quah decomposition.

\footnotetext{
${ }^{1}$ Artículo resultado de la investigación "Choques transitorios y de largo plazo en el Producto Interno Bruto (PIB) del Meta: un modelo de vectores autorregresivos estructurales”, financiado por la Universidad de los Llanos, en la primera convocatoria del año 2015.
} 


\section{Introducción}

Las fluctuaciones del Producto Interno Bruto (PIB) y los precios son tema de estudio recurrente dada su importancia en la economía, y su relación con los choques de oferta y de demanda de corto y largo plazo es analizado por un número importante de analistas en economías desarrolladas, menos en países subdesarrollados. La observación del impacto de los choques, sean de corto o largo plazo, requieren más atención, así como su persistencia en épocas estables o en crisis, por tanto, la investigación es importante para dar respuesta apropiada de una política que suavice los efectos de los choques transitorios sobre la economía y promover tasas de crecimiento sostenidas de la producción y estabilidad de precios. Como lo señala Lucas (1981), se deben conocer las características de las fluctuaciones macroeconómicas a corto plazo como un primer paso importante en el diseño de políticas de estabilización apropiadas.

En este sentido, es necesario conocer la influencia de las distorsiones económicas sobre el crecimiento y los precios, y, específicamente, este artículo propone conocer el impacto de los choques transitorios y de largo plazo en el departamento del Meta en Colombia.

Con una población de 906.805 habitantes, localizado entre el Piedemonte llanero y los Llanos orientales, con extensión de 85.779 kilómetros cuadrados, el departamento del Meta es el cuarto por tamaño en el país.

Desde 1990, y gracias a la apertura económica, su economía se ha expuesto a la dinámica de la economía mundial, la cual ha influenciado algunas de las variables más importantes, la producción y los precios. Algunos acontecimientos han intervenido más en la economía, como la crisis financiera, la crisis hipotecaria $y$, más recientemente, la crisis del petróleo. Gracias a la dinámica petrolera, la parti- cipación de las regalías en los ingresos del departamento entre 2008 y 2011 aportaron más del $50 \%$, pero a partir del 2012 con su reforma, la participación disminuyó hasta llegar al 14,4\% en el 2014, lo cual es causa de la reducción de la capacidad de gasto.

Para conocer si las crisis mencionadas influyeron en la dinámica económica del departamento del Meta, se utiliza un modelo de vectores autorregresivos estructurales (VAR) con el enfoque de Blanchard y Quah (1989). El modelo plantea que en el largo plazo el PIB es afectado solo por choques de oferta, y a corto plazo por choques de demanda; por su parte, los precios son influenciados a largo plazo por la oferta y demanda. Esto permite obtener los factores de corto y largo plazo del PIB. A diferencia de quienes trabajan este modelo, cuando usan el deflactor del PIB como variable representativa de los precios (Pérez, 2003), en esta investigación se utiliza el índice de precios al consumidor (IPC) porque, además de su disponibilidad, evita la correlación serial entre choques de demanda y oferta. Los resultados presentados coadyuvan a disminuir los niveles de incertidumbre a quienes toman decisiones de política económica porque ilustran sobre el impacto y la causalidad de los choques de oferta y demanda.

La estructura del artículo es la siguiente: la primera parte presenta una revisión de literatura, la segunda parte explica brevemente la metodología VAR, la tercera parte muestra un análisis empírico y los resultados y, por último, se discuten las principales conclusiones de la investigación.

\section{Revisión de literatura}

Inicialmente se utilizaron métodos univariados para descubrir las fuentes de los ciclos de las economías de los países desarrollados, descomponiendo los choques en permanentes y transitorios en las series de tiempo con énfasis en el estudio del comportamiento estocástico del producto. 
Nelson y Plosser (1982) utilizaron una serie temporal histórica de Estados Unidos y no rechazaron la hipótesis de que estas series son procesos estocásticos no estacionarios con ninguna propensión a volver a una línea de tendencia.

Otros teóricos afirmaron que las fluctuaciones de la producción no son desviaciones temporales; Campbell y Mankiw (1987) encontraron que un cambio inesperado en el Producto Nacional Bruto (PNB) real de uno por ciento debe cambiar de previsión en más del uno por ciento durante un largo horizonte.

Enfoques multivariados se utilizaron para examinar la importancia de los choques permanentes y transitorios. Los métodos multivariantes son generalmente preferibles porque emplean información de varias series de tiempo y maneja los choques empíricos de manera independiente, lo cual permite una interpretación estructural. Un enfoque multivariante es usado por Shapiro y Watson (1988) con modelo de autorregresión y muestra que las fluctuaciones de la producción son resultados de una mezcla de choques: de origen tecnológico, precios del petróleo y cambios en la oferta de trabajo, entre otros.

También usan el enfoque multivariado Bullard y Keating (1995) con el método de vectores autorregresivos estructurales (VAR) propuesto por Blanchard y Quah (1989) en un modelo de oferta y demanda agregada para analizar la relación entre la inflación y la producción real en 27 países; encontraron que un shock permanente en la inflación no está asociado con variaciones permanentes en el nivel de producción real. Keating y Nye (1998) estimaron la relación de largo plazo entre la inflación y la producción en las economías de posguerra con la metodología de VAR. Encontraron que los choques de producción permanente mueven el nivel de precios en la dirección opuesta, mientras los choques de producción temporales causan que el nivel de precios se mueva en la misma dirección.
Los estudios que incluyen el análisis de Blanchard y Quah y VAR estructurales (1989) utilizan un modelo de vector autorregresivo para estimar las fluctuaciones del PNB y desempleo en Estados Unidos como resultado de perturbaciones con efectos de largo y corto plazo. Los autores tienen en consideración dos tipos de choques no relacionados entre ellos y son diferenciables a corto y largo plazo. Exponen que el producto real se distingue entre componentes permanentes y transitorios, y muestran que el nivel de precios es afectado por choques de oferta y demanda. Suponen que al restringir uno de los choques por no tener efectos en alguna de las variables con independencia de los residuos, se conocen los errores del vector autorregresivo.

También la metodología de VAR expuesta por Blanchard y Quah (1989) es utilizada para conocer los efectos de los choques de demanda y oferta, la dinámica de los ciclos económicos, análisis de mercados financieros (Lustig y Verdelhan, 2011) y decisiones de política monetaria (Kaminska, 2008). Se ha utilizado el VAR para observar el impacto de los precios del petróleo de manera directa e indirecta con vectores autorregresivos y se muestra que existe evidencia entre el aumento de los precios petroleros y el crecimiento económico en Colombia como exportador de petróleo, pero el impacto es negativo si se trata de las exportaciones sectoriales por la revaluación de la moneda (Jiménez, 2010). Autores que estudian los choques de los precios del petróleo sobre el déficit en cuenta corriente mediante la metodología de VAR son Chuku, Sam y Effiong (2011).

Varios son los mecanismos identificados con la transmisión de choques de demanda y oferta sobre la economía. Estudios sobre el tema fueron realizados por Cashin, Mohaddes, Raissi y Raissi (2012), quienes diferencian los impactos entre positivos y negativos sobre la oferta y la demanda a corto y largo plazo. Choques en el PIB regional son tratados por Asongu (2013) y Bonilla (2016). 
Una manera de identificar los choques transitorios y de largo plazo en el PIB es asociar estos con las perturbaciones producidas por las crisis financieras y los precios del petróleo. Por el lado de las crisis financieras, según Gaona (2009), el shock financiero de 2008 afectó a las economías del mundo, y en Colombia el impacto de la crisis ocasionó que el PIB pasara de crecer $7,5 \%$ en 2007 a $2,5 \%$ en 2008 y a $0,36 \%$ en el 2009. El PIB del Meta también decayó en la época al igual que en Colombia.

El impacto de los últimos choques internacionales financieros sobre la economía es estudiado tomando como bases las crisis de 1998-1999 y 2008-2009 por el Banco de la República. La institución señaló que los choques internacionales de 2008-2009 tuvieron mayor impacto sobre la economía colombiana que en 1998-1999, y en el caso de la crisis de fin de siglo, el impacto se potenció con tres factores locales: la situación política, la violencia y las políticas fiscales. (Echavarría, Gonzáles, López y Rodríguez, 2012, p. 1)

Toledo (2004) aplicó un modelo VAR para resolver inquietudes con respecto a la aplicación de los VAR en Puerto Rico entre 1950 y 2002, y reafirmó la hipótesis de Keating y Nye (1998) cuando observó que los impulsos de oferta son más importantes para la dinámica del PNB a largo plazo, y a su vez, los impulsos de la demanda influyen en la dinámica a corto plazo; asimismo, agregó que las fluctuaciones de los precios dependen de los shocks de la demanda agregada. Mientras Toledo reafirma los efectos de la demanda y la oferta según la interpretación de Keating y Nye, el mismo Keating (2013) se pregunta: ¿qué se puede aprender de Blanchard y Quah sobre la descomposición de la producción si la demanda agregada no puede ser neutral a largo plazo? Así que analizó los impulsos respuesta de la producción frente a un shock permanente antes de la Primera Guerra Mundial y después de la Segunda Guerra Mundial. Llegó a dos conclusiones: la primera es que la evidencia empírica adicional apoya la hipótesis de que un aumento (disminución) de la demanda agregada tuvo un efecto positivo (negativo) permanente sobre la producción en las economías anteriores a la Primera Guerra Mundial; la segunda conclusión es que los éxitos aparentes con las descomposiciones de Blanchard y Quah en los datos de posguerra no significan necesariamente que las no neutralidades no sean importantes para ese período.

Mayorga, Torres y Méndez (1997) analizaron el efecto de los choques de origen externo sobre la producción y el tipo de cambio en Costa Rica con la técnica de VAR en datos trimestrales del periodo 1982 a 1995. Indicaron que los VAR incorporan la Función Impulso-Respuesta (FIR) y la Descomposición de la Variancia (DV). Encontraron un efecto simétrico de los choques, es decir, influyen con igual magnitud y duración, o sea, una caída en la actividad económica mundial origina un descenso permanente en la producción nacional y los términos de intercambio.

Roach (2014) usó modelos de vectores autorregresivos estructurales para conocer el efecto de los choques del precio del petróleo en variables macroeconómicas de Jamaica entre 1997 y 2012 , y observó que los choques del precio del petróleo no tienen efecto permanente sobre la economía de Jamaica. La autora menciona a un destacado estudioso quien relaciona los choques de precios del petróleo y las variables macroeconómicas: Hamilton (como se citó en Roach, 2014), en su influyente documento, destacó que un incremento brusco en los precios del petróleo crudo fue un precursor de siete de las ocho recesiones de la posguerra en Estados Unidos, en particular durante el periodo 1948-1972, basado en la significancia estadística de la correlación entre los choques petroleros y el crecimiento del PIB real. 
Un estudio del impacto de los precios del petróleo sobre el crecimiento económico en la economía colombiana entre 1982-2013 fue realizado por Gonzáles (2015), quien utiliza el modelo VAR con las funciones impulso-respuesta para sostener "la evidencia internacional" de que el principal medio de transmisión de los choques de precios del petróleo es el consumo privado, pero no encontró resultados que soporten la hipótesis. Sin embargo, sí encuentra relación entre consumo privado y producción.

\section{Metodología}

Se trabaja en el VAR basado en el enfoque de Blanchard y Quah (1989) y aplicado al contexto del departamento del Meta porque facilita asociar variables económicas al restringirlas en la identificación de las relaciones estructurales para conocer el impacto de largo plazo de los choques de oferta sobre el producto, suponiendo que los precios no tienen impacto inmediato sobre el PIB.

Los datos del PIB del departamento del Meta son obtenidos del Departamento Administrativo Nacional de Estadística (DANE) del año 2000 al 2015 con $\mathrm{n}=26$ observaciones y se toman las variaciones del Índice de Precios al Consumidor (IPC) de Villavicencio como variable proxy para medir el nivel general de precios y su variación en el departamento del Meta, considerando que Villavicencio es la capital del departamento. El tamaño de la muestra no es adverso, aun comparándolo con estudios que utilizan muestras de tamaño parecido, por ejemplo, Bullard y Keating (1995) utilizan 29 observaciones. Además, al calcular la matriz de varianzas y covarianzas de los errores, dado el tamaño de la muestra, se utilizará un ajuste de los grados de libertad, es decir, $1=1 /(\mathrm{T}-)$, en lugar del divisor para muestras grandes $1 / \mathrm{T}$, donde es el número medio de parámetros en la forma funcional para $\mathrm{y}_{\mathrm{t}}$ sobre las $\mathrm{K}$ ecuaciones. Esta muestra abarca tres periodos de crisis: el primer periodo es la crisis financiera entre 1998 y 1999 (Torres, 2011), en esta fecha algunas de las más importantes entidades financieras se quebraron y fue causado especialmente por los deudores de vivienda, quienes no podían asumir sus elevadas deudas; el segundo periodo marcado por la crisis del mercado hipotecario en Estados Unidos, que contagió a Colombia en 2008 y 2009 (ANIF, 2011), y el último corresponde a la caída del precio del petróleo en el 2015 (Saavedra, 2016). Para modelar los impactos de estas crisis se crean variables cualitativas dummy, para captar el impacto dinámico de las crisis sobre el PIB y la inflación. La variable dummy adopta el valor de 0 para los periodos de crisis y de 1 para los demás periodos.

La implementación del VAR bivariado, como lo trabajan Blanchard y Quah (1989), partirá de la comprobación que el PIB y los precios son variables integradas de orden uno, I(1). La primera diferencia del logaritmo del PIB muestra el crecimiento del PIB y la primera diferencia del logaritmo del índice de precios presenta como resultado la inflación, es decir, $\Delta y t$ у пt. De manera formalizada, la representación del enfoque de Blanchard y Quah para los vectores autorregresivos es:

$$
\begin{aligned}
\Delta \mathrm{yt} & =\sum_{i=0}^{\infty} c_{11}(\mathrm{i}) \varepsilon_{\Delta \mathrm{yt}-\mathrm{i}}+\sum_{i=0}^{\infty} c_{12}(\mathrm{i}) \varepsilon_{\pi \mathrm{t}-\mathrm{i}} \\
\pi \mathrm{t} & =\sum_{i=0}^{\infty} c_{21}(\mathrm{i}) \varepsilon_{\Delta \mathrm{yt}-\mathrm{i}}+\sum_{i=0}^{\infty} c_{22}(\mathrm{i}) \varepsilon_{\pi \mathrm{t}-\mathrm{i}}
\end{aligned}
$$

donde yt es la producción, nt son los precios y $c_{11}(\mathrm{i}) \mathrm{c}_{12}(\mathrm{i}), \mathrm{c}_{21}$ (i) y $\mathrm{c}_{22}$ (i) miden la respuesta de las secuencias completas del crecimiento del PIB y los precios. 
En el modelo estándar de oferta y demanda agregada, los choques permanentes al producto son causados por choques de oferta, mientras que los choques temporales provienen de choques de demanda (Keating y Nye, 1998). En el enfoque de Blanchard y Quah se supone que los choques asociados a la ecuación (1) son permanentes, mientras que los choques asociados a la ecuación (2) tienen un impacto temporal.

Al trabajar con el modelo de Blanchard $\&$ Quah, el PIB real se asocia con efectos de largo plazo y a corto plazo con los choques exógenos $\varepsilon_{\pi}$; para que esto suceda, el siguiente factor, proveniente de la ecuación (1) del crecimiento del producto, supone que los residuos presentan un efecto acumulado igual a cero:

$$
\sum_{i=0}^{\infty} c_{12}(\mathrm{i}) \varepsilon_{\pi \mathrm{t}-\mathrm{i}}=0
$$

Un inconveniente de los VAR estándar es que no reconoce el origen de los choques, este inconveniente se resuelve con los VAR estructurales (VAR). Según Watson (1994), el modelo VAR se identifica así:

$$
A_{o} y_{t}=A_{1} y_{t-1}+A_{2} y_{t-2}+\ldots+A_{p} y_{t-p}+\varepsilon_{t}(4)
$$

Donde A es un operador de rezago, $y t \mathrm{y}$ $y_{t-p}$ son series de tiempo escalar; si se le adicionan variables exógenas $X_{t}$, el modelo es: $y t=v+A 1 y t-1+\cdots+A p y t-p+B 0 x t+$ $B 1 x t-1+\cdots+B s x t-s+u t t \in\{-\infty, \infty\}(5)$

donde

$y t=(y 1 t, \ldots, y K t) 0$ is a $K \times 1$ es un vector aleatorio,

$A 1$ a través de $A p$ son $K \times K$ parámetros de matrices,

$x t$ es un $M \times 1$ vector de variables exógenas, $B 0$ a través de $B s$ es $K \times M$ matrices de coeficientes,

$v$ es un $K \times 1$ vector de parámetros, y

$u t$ es asumido para ser ruido blanco, que es $E(u t)=0, E(u t u 0 t)=\Sigma$, y $E(u t u 0 s)=0$ para

$t \neq s$
Este supuesto diferencia los componentes transitorios y permanentes del PIB. El VAR estructural para el análisis de corto y largo plazo es:

$$
A\left(I_{k}-A_{1} L-A_{2} L^{2}-\ldots-A_{p} L^{p}\right) y_{t}=A \varepsilon_{t}=B e_{t}
$$

El operador de rezago se denota por $L$, A, $B$ y $A_{1}, \ldots, A_{p}$, son matrices de orden $K \times K, \varepsilon_{t}$ es un vector que representa innovaciones cuando $\varepsilon_{t} \mathrm{~N}\left(0, \sum\right)$, y $E\left(\varepsilon_{t} \varepsilon_{s}{ }^{\prime}\right)=0_{K}$ para todo $s \neq t$ y $e_{t}$ es un vector $K \times 1$ de residuos ortogonalizados, es decir, $\mathrm{e}_{t} \mathrm{~A}\left(0, \sum\right) \mathrm{y}$ $E\left(e_{t} e_{s}\right)=0_{K}$ para todo $s \neq t$. Las restricciones de las matrices $A$ y $B$, que son singulares, permiten la identificación.

Para determinar el VAR de largo plazo, se calcula la siguiente ecuación, como la hacen Misas y López (1999):

$$
\begin{aligned}
& y t={ }^{-1} B e_{t} \\
& y_{t}=C e_{t}
\end{aligned}
$$

Donde $C={ }^{-1} B$ y $\sum=B B^{\prime}$.

En la matriz $C$ se aplican las restricciones de largo plazo del modelo VAR. Las restricciones en el modelo bivariado señalan que los choques de demanda tienen impacto de corto plazo sobre el PIB, así mismo, los choques de oferta tienen efecto de largo plazo; por otro lado, los precios son influenciados por los choques de oferta y demanda. La matriz $C$ incorpora las restricciones asi:

$$
C=\left[\begin{array}{ll}
\cdot & 0 \\
\cdot & \cdot
\end{array}\right]
$$

Es decir, los choques de demanda presentan un impacto de 0 sobre el producto de largo plazo. Está asociado a la explicación de que el aumento de la demanda a largo plazo no influye en la producción, mas bien, esta depende del aumento de la capacidad productiva. Por último, se calcula la influencia de los choques con los multiplicadores dinámicos después de encontrar el VAR para conocer la diferencia de la sensibilidad del PIB y los precios frente a las crisis. 


\section{Resultados}

Se espera que tanto el PIB como los precios sean variables no estacionarias, integradas de orden uno $I(1)$, para obtener los componentes de corto y largo plazo del PIB y los precios, por lo cual se analizan las variables PIB y precios para conocer su estacionariedad.

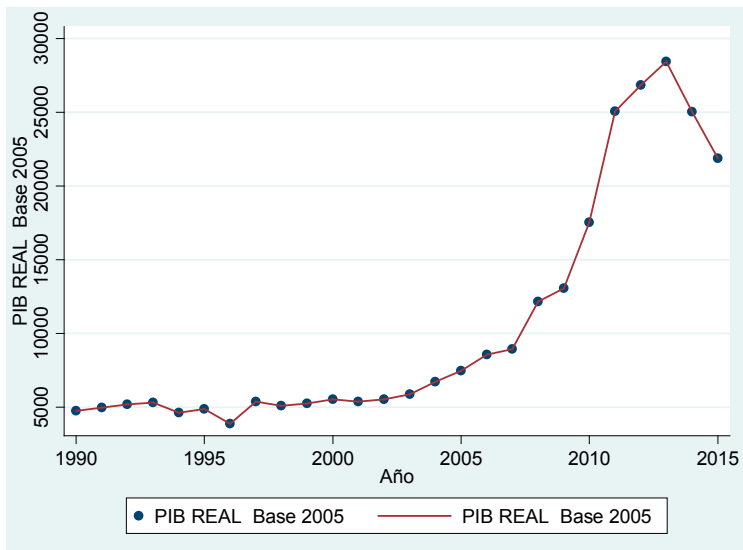

Fig. 1a. Gráfico de tendencia del PIB real. base 2005 del Meta, 1990-2015.

Fuente: Elaboración propia con base en DANE, 2016.

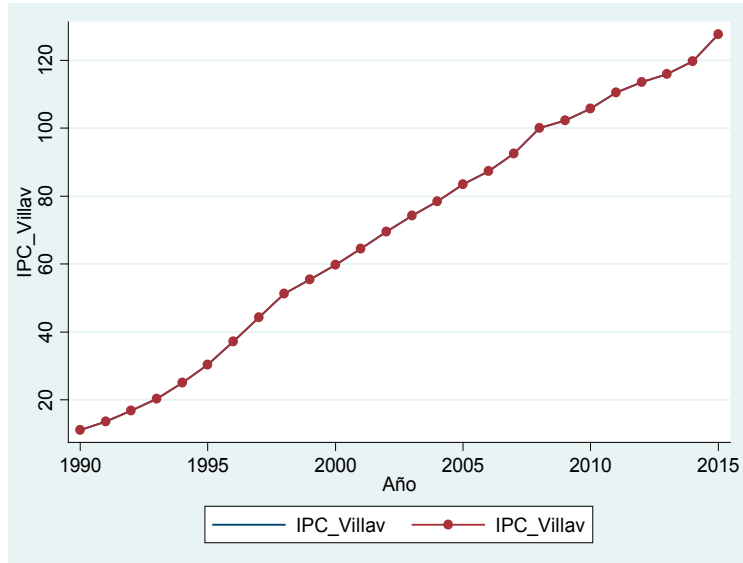

Fig. 1b. Gráfico de tendencia del Índice año base 2005 del Meta, 1990-2015.de Precios al Consumidor en Villavicencio.

Fuente: Elaboración propia con base en DANE, 2016.

Las figuras 1a y $1 \mathrm{~b}$ muestran que las dos variables en niveles tienen un comportamiento no estacionario, lo que pue- de indicar la existencia de algún tipo de tendencia determinística o estocástica. Debido a esto, se verifica la no estacionariedad de los datos con las pruebas Dickey Fuller Aumentada (ADF) y Phillips Perron. La prueba ADF de raíz unitaria para el PIB se corre con una constante y un término de tendencia para determinar si existe correlación serial (Anexo A). La prueba ADF indica que no puede rechazarse la hipótesis nula de raíz unitaria, lo que sugiere que la variable del PIB en niveles no es estacionaria. Esta información es favorable debido a que se puede extraer el componente de largo plazo de esta serie.

Usando la primera diferencia logarítmica del PIB, puede verificarse que el crecimiento del PIB (Dlpibreal) es una variable estacionaria y altamente significativa (Anexo B). El examen de estacionariedad del PIB usando la prueba ADF permite estimar que el nivel del PIB es una variable integrada de orden uno, $I(1)$, o, de manera alternativa, que el crecimiento del PIB es una variable estacionaria o $I(0)$. Por su parte, en el gráfico de los precios se observa en principio una tendencia positiva marcada, por lo que es necesario conocer si esa tendencia tiene un origen estocástico o determinista.

En el caso de los precios, denotados como IPC_Villav, se observa que no se les puede rechazar la hipótesis nula de raíz unitaria a un nivel de significancia del $5 \%$, es decir, la variable no es estacionaria (Anexo C). En lo relativo a la inflación, se obtiene que la primera diferencia de los precios es una variable estacionaria, tal como lo sugiere el rechazo de la hipótesis de raíz unitaria (Anexo D). Es decir, los precios son una variable integrada de primer orden $I(1)$, y la inflación es una variable integrada de orden cero o $I(0)$.

El componente de tendencia deterministica es significativo. En varios países de América Latina, el PIB tiene tendencia 
determinista, y, por consiguiente, las variables se han corrido de forma determinística. Mejía y Hernández (1998) indican que en México, el PIB tiene "tendencia determinista $\mathrm{y}$, por tanto, los choques son transitorios" (p. 457); en Chile (Idrovo, 2010); en Cuba, Vidal y Fundadora (2004) muestran que "los componentes que describen la evolución de una serie de tiempo (tendencia, estacionalidad, ciclo y componente irregular) se han modelado tradicionalmente de forma determinista" (p. 6).
Vectores autorregresivos (VAR). Dado que se ha determinado el orden de cointegración del PIB y los precios, se especifica un modelo de vectores autorregresivos. Se selecciona el crecimiento del PIB (Dlpibreal) y el crecimiento de los precios (dlIPC_Villav) porque el modelo requiere que las variables sean estacionarias. El primer paso es determinar el orden del VAR (Anexo E); las pruebas de Akaike y de Hannan-Quinn indican que la mejor opción son dos rezagos (Tabla 1):

TABLA 1

Vector de autorregresión.

\begin{tabular}{|c|c|c|c|c|c|c|}
\hline Sample: & -2015 & & & No. $\circ$ & obs & 23 \\
\hline Log likelihood & 49.45101 & & & AIC & & $=-2.908783$ \\
\hline FPE & .0001986 & & & $\mathrm{HQIC}$ & & $=-2.710123$ \\
\hline Det (Sigma_ml) & .0000465 & & & SBIC & & $=-2.118874$ \\
\hline Equation & Parms & RMSE & $R-s q$ & chi2 & P>chi2 & \\
\hline Dlpibreal & 8 & .151928 & 0.3213 & 10.88726 & 0.1436 & \\
\hline dlIPC_Villav & 8 & .068919 & 0.8249 & 108.3502 & 0.0000 & \\
\hline
\end{tabular}

\begin{tabular}{|c|c|c|c|c|c|c|}
\hline & Coef. & Std. Err. & z & $P>|z|$ & [95\% Conf. & Interval] \\
\hline \multicolumn{7}{|l|}{$\begin{array}{l}\text { Dlpibreal } \\
\text { Dlpibreal }\end{array}$} \\
\hline L1. & -.14437 & .2061947 & -0.70 & 0.484 & -.5485042 & .2597641 \\
\hline L2. & .4473571 & .2284035 & 1.96 & 0.050 & -.0003056 & .8950197 \\
\hline \multicolumn{7}{|l|}{ dIIPC_Villav } \\
\hline L1. & -.1550207 & .2779819 & -0.56 & 0.577 & -.6998553 & .3898139 \\
\hline L2. & .0046755 & .2219052 & 0.02 & 0.983 & -.4302507 & .4396017 \\
\hline dfinanciera & -.1042065 & .1979433 & -0.53 & 0.599 & -.4921683 & .2837552 \\
\hline dhipoteca & .1313888 & .0935213 & 1.40 & 0.160 & -.0519096 & .3146872 \\
\hline dpetroleo & -.2293237 & .138129 & -1.66 & 0.097 & -.5000516 & .0414041 \\
\hline _cons & .055039 & .0598588 & 0.92 & 0.358 & -.0622821 & .1723601 \\
\hline \multicolumn{7}{|l|}{$\begin{array}{r}\text { dlIPC_Villav } \\
\text { Dlpibreal }\end{array}$} \\
\hline$\quad$ L1. & .1761146 & .0935357 & 1.88 & 0.060 & -.0072119 & .3594412 \\
\hline L2. & -.3609634 & .1036102 & -3.48 & 0.000 & -.5640357 & -.157891 \\
\hline \multicolumn{7}{|l|}{ dlIPC_Villav } \\
\hline L1. & .5588603 & .1261004 & 4.43 & 0.000 & .311708 & .8060125 \\
\hline L2. & .2787181 & .1006624 & 2.77 & 0.006 & .0814234 & .4760128 \\
\hline dfinanciera & -.5479484 & .0897926 & -6.10 & 0.000 & -.7239387 & -.3719581 \\
\hline dhipoteca & -.0503892 & .0424239 & -1.19 & 0.235 & -.1335385 & .0327601 \\
\hline dpetroleo & .0336282 & .0626592 & 0.54 & 0.591 & -.0891816 & .1564379 \\
\hline _cons & .0492336 & .0271536 & 1.81 & 0.070 & -.0039865 & .1024537 \\
\hline
\end{tabular}

Fuente: Elaboración propia con base en DANE, 2016. 
En la ecuación del crecimiento del PIB no se observa impacto sobre este. Por su parte, la ecuación de la inflación muestra que la afecta positivamente su primer rezago y segundo rezago, por el contrario, el PIB no la afecta. Solo la crisis financiera tiene un impacto significativo y negativo sobre la inflación.

Es relevante identificar que los residuos y los residuos al cuadrado se distribuyen de manera independiente, en otras palabras, que no tienen correlación serial, para lo cual se realiza una prueba con el multiplicador de Lagrange (LM) sobre los residuos (Anexo $\mathrm{F}$ ).

No se presenta evidencia de que existe autocorrelación serial de los residuos, excepto en el rezago del año 15 con una probabilidad de $90 \%$. A pesar de lo anterior, se considera que no es un problema grave y puede estar asociado a los ciclos económicos. Sumado a la prueba de correlación serial de los residuos, se examina el comportamiento de los residuos de cada ecuación para reforzar que los residuos y los residuos al cuadrado no tienen correlación serial o son ruido blanco con pruebas de Portmanteu (Castle y Hendry, 2010) (Anexo G).

Con respecto a los residuos del PIB, se puede afirmar que existe el $84,30 \%$ de probabilidad y, por consiguiente, no se puede rechazar la hipótesis nula de que los residuos del PIB son independientes, por tanto, la variable residual es ruido blanco y no estacionaria. La misma interpretación se aplica a los residuos del precio y los residuos al cuadrado.

El VAR debe cumplir con las condiciones de estabilidad, y según Brugger (2010), "con esto se asegura que ante choques de corto plazo las variables regresen a su trayectoria de equilibrio de largo plazo. Si no se diera el caso, se tendría un modelo explosivo, lo que no tendría sentido económico" (p. 161). En esencia, puede suceder que, ante falta de estabilidad del
VAR, el futuro lejano puede influir en el presente y el modelo no se ajustaría rápidamente frente a choques temporales. Para la estabilidad se requiere que las raíces se encuentren en el círculo unitario, teniendo en cuenta que desde el comienzo se supone que los promedios móviles del VAR presentan un sistema estable.

El módulo de los valores propios es menor a uno (Anexo $\mathrm{H}$ ), es decir, los valores propios se ubican dentro del círculo unitario (figura 2), por tanto, el modelo VAR cumple la condición de estabilidad.

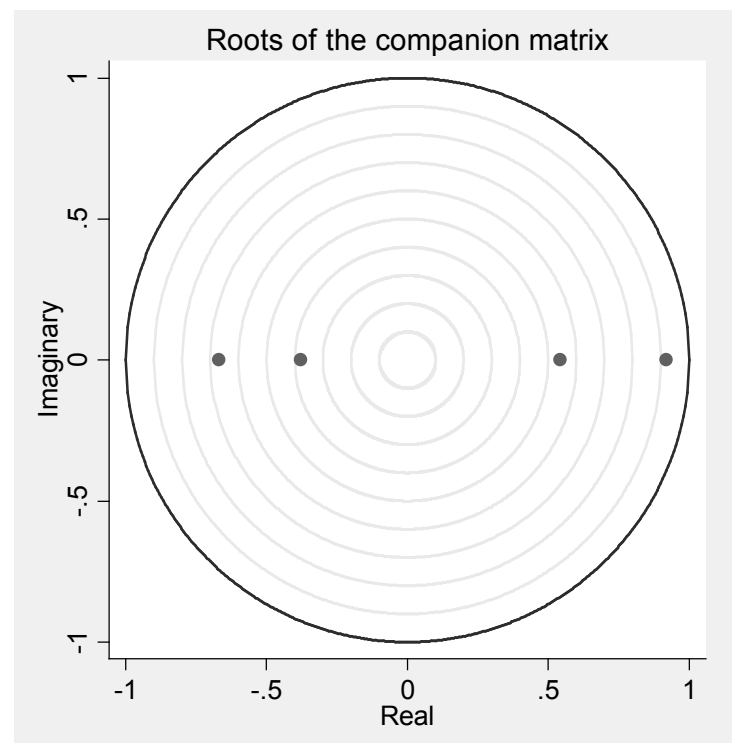

Fig. 2. Gráfico de raíces del VAR.

Fuente: Elaboración propia con base en DANE, 2016.

Funciones de impulso respuesta. Una vez estimado el VAR para visualizar los periodos de crisis con las variables exógenas, se generan los multiplicadores dinámicos para visualizar la sensibilidad diferenciada de las crisis sobre el crecimiento económico y la inflación. Los multiplicadores se grafican en la figura 3a y $3 b$, y se observa que el shock negativo en el sistema financiero sobre el PIB inicialmente fue de $32,99 \%$, lo siguió una caída en el crecimiento del PIB real del año 1 en 29,40\%, en el año 2 de $27,81 \%$ $\mathrm{y}$ se mantiene en un nivel estacionario. 
El shock financiero sobre la inflación en el año 0 fue de 7,9\%, en el año 1 disminuyó en 11,35 \% y en el año 2 en 16,45\% y va en aumento el impacto de tal forma que, en el año 16, la caída de los precios es del 59,82\% (Fig. 3a). Badel (2002) analizó con un modelo de ciclos crediticios de Kiyotaki y Moore esta crisis en Colombia, y explicó que, en la época, los flujos de capital externo disminuyeron, se desaceleró el crédito y, por consiguiente, el precio de los activos decayó, y dado que los deudores no tenían cómo pagar, ocasionó adición al recorte crediticio. El autor consideró que la recuperación es lenta y tomará 20 años por la dinámica entre los bajos precios de los activos y el crédito. En el departamento del Meta, un año después de que se presentó la crisis financiera en 1998, ocurrió la única disminución en la inflación durante el periodo de estudio con $25,16 \%$. Esta cifra y la posterior desaceleración de la inflación indican que la restricción de crédito afectó significativamente el nivel de precios en el departamento.

En la crisis hipotecaria, el shock en el año 0 sobre el PIB fue de una disminución del 14,66\%; seguido, en el año 1, de $15,52 \%$; y en el año 2 aumentó $24,73 \%$; $\mathrm{y}$, hasta el año 10, presenta un ciclo ligeramente cíclico que oscila entre el $25 \%$ y $22 \%$, después desaparece el efecto. Por el lado de la inflación, se presentó un shock inicial negativo de $0,55 \%$, en el año 1 de $1,52 \%$, en el año 2 de $2,24 \%$, y en adelante, la disminución de la inflación es leve (figura $3 b$ ). El efecto de la crisis hipotecaria sobre el PIB real y los precios es menor que las otras crisis.

Inicialmente, un shock negativo de los precios del petróleo ocasiona un efecto importante en el año 2 , y a partir del año 5 , el efecto se va amortiguando. Con relación a la influencia del petróleo sobre la inflación, aunque positivo, no es significativo (figura 3c). Posiblemente,

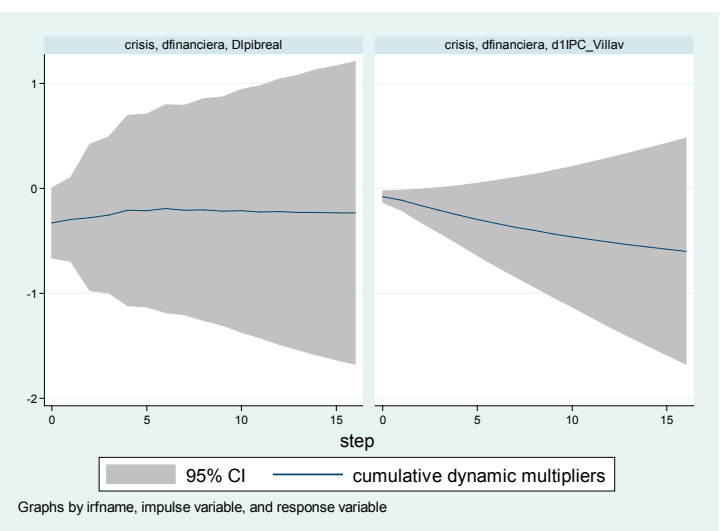

Fig. 3a. Gráfico de impulso-respuesta de la crisis financiera.

Fuente: Elaboración propia con base en DANE, 2016.

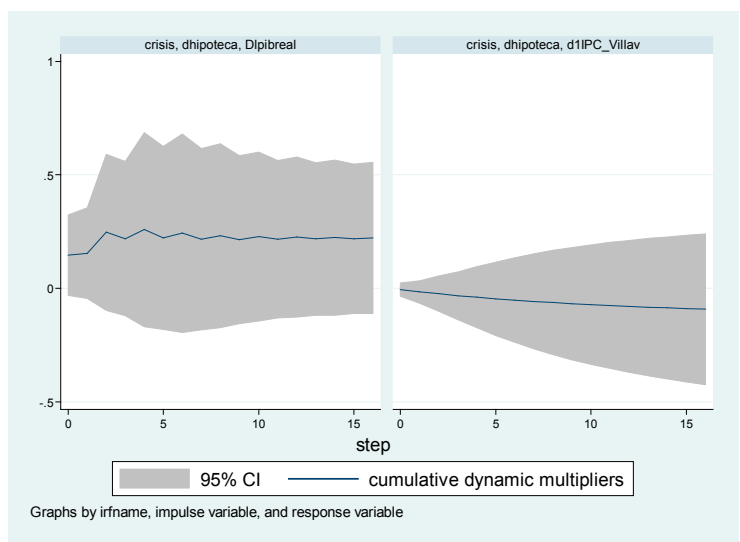

Fig. 3b. Gráfico de impulso-respuesta de la crisis hipotecaria.

Fuente: Elaboración propia con base en DANE, 2016.

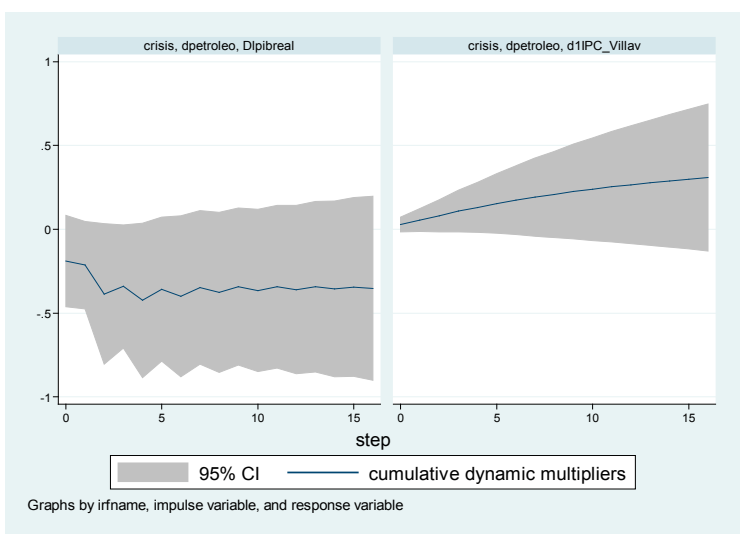

Fig. 3c. Gráfico de impulso-respuesta de la crisis del petróleo.

Fuente: Elaboración propia con base en DANE, 2016. 
el registro de la poca influencia de la crisis del petróleo sobre las variables de estudio se debe a que la crisis aparece recientemente, en el año 2015, por tanto, el periodo transcurrido no es suficiente para medir su impacto. En general, las crisis financieras y del petróleo impactan negativamente en la producción, y únicamente el shock financiero impacta significativamente sobre la inflación.

Vectores autorregresivos estructurales (VAR). Los VAR adicionan información a los choques porque permiten precisar el orígen de estos. Para esto, es preciso extraer los componentes de corto y largo plazo del producto al relacionar el choque exógeno de los precios con efectos de corto plazo sobre el producto (Keating y Nye, 1998). Según la teoría económica, el PIB se relaciona con choques de oferta de largo plazo, y a corto plazo se relaciona con choques de demanda, mientras que los precios se relacionan con los choques de oferta y demanda de largo plazo.

El vector de autorregresión estructural resultante muestra los coeficientes que estiman los efectos de choques de oferta y de demanda en el largo plazo (Tabla 2). Las restricciones conllevan a manejar dos supuestos: i) el PIB real es afectado por choques de oferta de largo plazo y los choques de demanda de corto plazo, y ii) los precios son afectados en el largo plazo por los choques de demanda y oferta. Estos supuestos son tomados del método de Blanchard y Quah (1989), que atañe a la rigidez nominal los efectos de corto plazo de la demanda sobre la producción, pero estos desaparecen con el tiempo. En el largo plazo, la oferta influencia la producción. De otra parte, los disturbios de la demanda y la oferta afectan los precios.

Los choques de oferta tienen un impacto de largo plazo positivo y significativo sobre el PIB real del departamento del Meta con un coeficiente de 0,248 y un impacto de largo plazo negativo y significativo sobre los precios con un coeficiente de -0,401; asimismo, los precios reaccionan positiva y significativamente a los choques de demanda en el largo plazo. Dada la restricción, el PIB no es afectado por la demanda a largo plazo, por tanto, su coeficiente es 0 .

TABLA 2

Vector de autorregresión estructural (VAR).

( 1 ) [c_1_2] cons $=0$

Sample: 1993 - 2015

Exactly identified model

No. of obs $=23$

Log likelihood $=49.45101$

\begin{tabular}{|c|c|c|c|c|c|c|}
\hline & Coef. & Std. Err. & z & $\mathrm{P}>|\mathrm{z}|$ & [95\% Conf. & Interval] \\
\hline / ${ }_{-}{ }^{1}{ }_{-} 1$ & .2483734 & .0366207 & 6.78 & 0.000 & .1765982 & .3201485 \\
\hline / $c_{-}{ }^{2}{ }_{-}{ }^{1}$ & -.4013795 & .0894144 & -4.49 & 0.000 & -.5766284 & -.2261305 \\
\hline$/ \mathrm{C}_{-}{ }^{1} \mathrm{~B}^{2}$ & 0 & (omitted) & & & & \\
\hline$/ c_{-}{ }^{2}{ }^{2} 2$ & .3214509 & .0473953 & 6.78 & 0.000 & .2285577 & .4143441 \\
\hline
\end{tabular}

Fuente: Elaboración propia con base en DANE, 2016. 


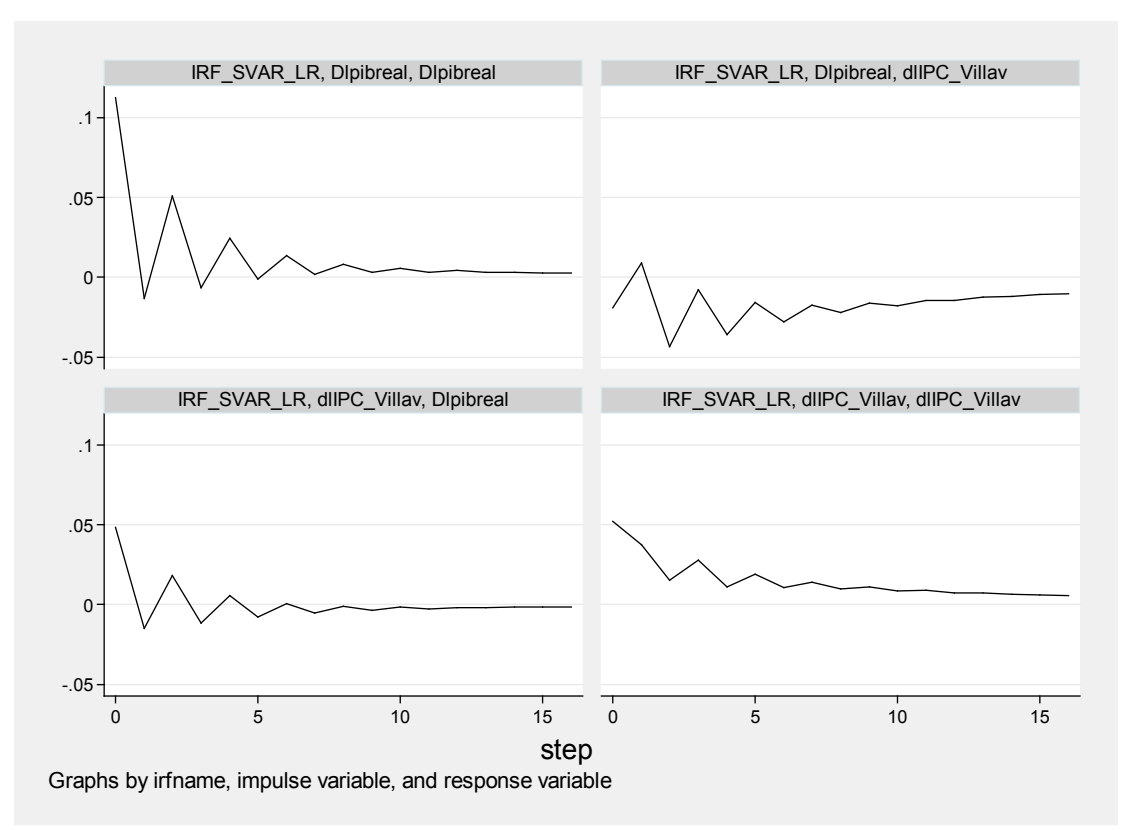

Fig. 4a, b, c y d. Gráfico de impulso-respuesta de la VAR.

Fuente: Elaboración propia con base en DANE, 2016.

La función de impulso-respuesta del VAR, graficada en la figura 4a, muestra que el impacto de los choques de largo plazo de la oferta sobre el crecimiento del producto es amplio durante los primeros cinco años, después desaparece abruptamente. En la figura $4 \mathrm{~b}$ se observa que la inflación reacciona negativamente al choque de largo plazo de la oferta en los primeros seis años, y se atenúa en los siguientes años. Los choques de demanda tienen inicialmente una influencia cíclica sobre el crecimiento del producto en los cuatro primeros años y en adelante se desvanece el ciclo (figura 4c). La figura $4 \mathrm{~d}$ visualiza un impacto inicial positivo de los choques de demanda sobre la inflación, y a partir del año 6 se desvanece. En resumen, el crecimiento del PIB real del departamento del Meta y la inflación son influenciados significativamente por los choques de oferta, mientras la producción y la inflación reaccionan levemente a choques de demanda.

\section{Conclusiones}

Al analizar los impactos de largo plazo de los choques de oferta sobre el PIB y los precios en el departamento del Meta entre 1990-2015, se ilustra que el PIB y los precios tienen raíz unitaria, lo cual permite obtener los elementos de largo plazo de las variables, requisito necesario para adelantar el procedimiento de Keating y Nye (1998), quienes establecen una curva de oferta agregada de largo plazo, vertical e independiente de la demanda, donde su impacto es de largo plazo sobre el producto; mientras la demanda tiene influencia a corto plazo sobre el producto. Esta restricción permite aislar el elemento de largo plazo del PIB del departamento del Meta. Partiendo de este análisis, se observa que los choques de oferta de largo plazo asumen un impacto positivo en el producto y ocasionan la caída de los precios. A su vez, los choques de demanda ejercen un impacto leve y positivo sobre los precios y poco significativo sobre la producción. 
De las crisis, la única que afecta a algunas de las variables de estudio, es la crisis financiera sobre los precios de forma negativa, y en vista de que en el modelo VAR los precios contienen el componente de oferta, se atribuye el origen de este choque a la contracción en la oferta. Como la política fiscal hasta antes de 1989 no tenía mayor efecto para reducir la inflación y, a partir de la crisis financiera de 1999 la inflación se redujo notablemente, bien podría asociarse la desaceleración del crecimiento de los precios a corto plazo con la crisis financiera, y a mediano plazo y largo plazo, además de la crisis, con la nueva política fiscal que entró en vigor en el 2000 que conllevó a establecer un régimen de inflación objetivo para alcanzar una inflación específica dentro de un tiempo determinado.

En la época, como resultado del ajuste fiscal en Villavicencio y la política de vivienda de interés social, el banco emisor señala que se reactivó la construcción después del periodo de la crisis financiera (Banco de la República, 2002, p. 3).

\section{Referencias}

ANIF (2011). El crédito hipotecario en Colombia: evaluación del impacto regulatorio pos-crisis. Bogotá: ANIF. Recuperado de: http://anif.co/sites/default/files/uploads/Anif- BID0511.pdf

Asongu, S. (2013). Nuevas empíricas de la dinámica de la política monetaria: datos procedentes de las zonas del franco CFA. Documento de trabajo de AGDI, No. WP/13/016. Gobernanza africana y el Instituto de Desarrollo (AGDI).

Badel, A. (2002). Choques financieros, precios de activos y recesión en Colombia. Desarrollo y sociedad, 49, 61-97.

Banco de la República (2002). Informe de coyuntura económica regional del departamento del Meta. Bogotá, D.C.: Banco de la República.
Blanchard, O. y Quah, D. (1989). The dynamics effects aggregate demand and supply disturbances. American Economic Review, 79(4), 655-673.

Bonilla, L. (2016). Choques externos y remesas internacionales en las regiones de Colombia. Documentos de trabajo sobre economía regional Núm. 250. Banco de la República.

Brugger, S. (2010). Capital especulativo y crisis bursátil en América Latina. México, D.F., México: Editorial Eumed.

Bullard, J. y Keting, J. (1995). The longrun relationship between inflation and output in postwar economies. Journal of Monetary Economics, 36, 477- 496.

Cashin, P., Mohaddes, K., Raissi, M. y Raissi, M. (2012). The Differential Effects of Oil Demand and Supply Shocks on the Global Economy. IMF working paper WP/12/253: 2-41.

Castle, J. y Hendry, D. (2010). A low-dimension portmanteau test for non-linearity. Journal of econometrics, 158, 231-245.

Campbell, J. Y. y Mankiw, N. G. (1987). Permanent and Transitory Components in Macroeconomic Fluctuations. American Economic Review, 77(2), pp. 111117.

Chuku, Ch., Sam, N. y Effiong, E. (2011). Oil Price Shocks and the Dynamics of Current Account Balances in Nigeria. OPEC Energy Review, 35(2), 119-139.

DANE (2016). Cuentas departamentales del Meta. Recuperado de: https://www. dane. gov.co/index.php/estadisticas-portema/cuentas-nacionales/cuentas-nacionales-departamentales

Echavarría, J. J, Gonzáles, A., López, E. y Rodríguez, N. (2012). Choques internacionales reales y financieros y su impacto sobre la economía colombiana. Borradores de Economía (728). Banco de la República. 
Gaona, M. D. (2009). Efectos de la crisis financiera internacional sobre el panorama fiscal. Informe de la Contraloría General de la República. Colombia.

Gonzáles, S. (2015). Impacto de los precios del petróleo sobre el crecimiento económico, evidencia para Colombia (19822013). [Tesis de maestría]. Maestría en Ciencias Económicas, Universidad Nacional de Colombia. Bogotá, Colombia. Recuperado de: http://www.bdigital. unal.edu.co/48291/

Idrovo, B. (2010). ¿Cuál es el crecimiento de largo plazo de la economía chilena? una respuesta formal para una antigua pregunta. Revista Cuadernos de Economía, 29(53), 31-55.

Jiménez, J. R. (2010). El impacto de los precios del petróleo sobre el crecimiento económico en Colombia. Revista de Economía del Rosario, 13(1), 75-116.

Keating, J. (2013). What do we learn from Blanchard and Quah decompositions of output if aggregate demand may not be long-run neutral? Journal of Macroeconomics, (38), 203-217. Recuperado de: http://www2.kuedu/ kuwpaper/2009P apers/201302.pdf

Keating J. y Nye, J. (1998). Permanent and Transitory Shocks in Real Output: Estimates from Nineteenth-Century and Postwar Economies. Journal of Money, Credit and Banking, 30(2), 231-251.

Kaminska, I. (2008). A no-arbitrage structural vector autorregresive model of the UK yield curve. Working paper No. 357. Bank of England.

Lucas, R. (1981). Studies in business cycle theory. Cambridge: Masachusetts Institute of Technology.

Lustig, H. y Verdelhan, A. (2011). The cross section of foreign currency risk premia and consumption growth risk: reply. The American Economic Review, 101(7), 3477-3500.
Mayorga, J., Torres, C. y Méndez, E. (1997). Efectos de los choques de origen externo sobre la producción y el tipo de cambio real de Costa Rica. Banco Central de Costa Rica.

Mejía, P. y Hernández, Z. (1998). Evolución del Producto Interno Bruto en México, ¿Declinación o Histéresis? Evidencia adicional. Revista Economía, Sociedad y Territorio, 1, 457-491. Recuperado de: http://www.redalyc.org/articulo. oa?id=11110302.

Misas, M. y López, E. (1999). El producto potencial en Colombia: Una estimación bajo VAR estructural. Serie cuadernos de investigación, (94).

Pérez, P. (2003). Fuentes de variabilidad en las principales economías departamentales. Investigaciones económicas, 27(3), 565-591.

Roach K. (2014). Un análisis estructural de los choques de precios del petróleo en la macroeconomía de Jamaica. Revista Monetaria, 36(2). 233-271.

Shapiro, M. y. Watson, M. (1988). Sources of Business Cycle Fluctuations. National Bureau of Economic Research Macroeconomics Annual, 111-48.

Toledo, W. (2004). Impulsos de demanda y oferta agregada y las fluctuaciones económicas en Puerto Rico de 1950 a 2002. Revista Economía, (19-20), 35-57.

Torres, A. (2011). La crisis colombiana de finales del siglo XX: ¿Un choque real o financiero? Perfil de coyuntura económica. Medellín: Universidad de Antioquia. Recuperado de: http://aprendeenlinea. udea.edu.co/revistas/index.php/coyuntura/article/view/13246/11872

Vidal, P. y Fundadora, A. (2004). Tendencia y ciclos en el Producto Interno Bruto de Cuba: Estimación con un Modelo Estructural Univariante de Series Temporales. 42 Aniversario de los Estudios Económicos en la Facultad de Economía de la Universidad de la Habana. La Habana. 


\section{Anexo A. Prueba de raiz unitaria para el PIB}

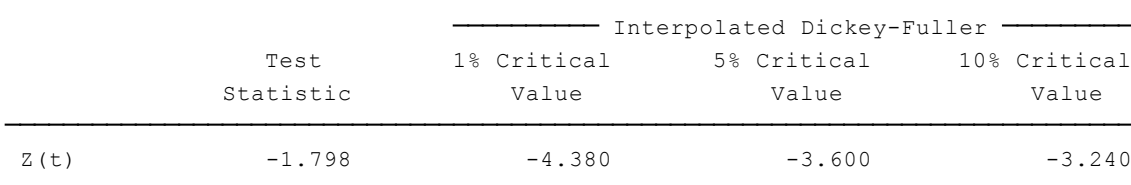

Mackinnon approximate $\mathrm{p}$-value for $\mathrm{Z}(\mathrm{t})=0.7059$

\begin{tabular}{|c|c|c|c|c|c|c|}
\hline D.lpibreal & Coef. & std. Err. & $t$ & $P>|t|$ & [95\% Conf. & Interval] \\
\hline \multicolumn{7}{|l|}{ lpibreal } \\
\hline L1. & -.1940068 & .1079192 & -1.80 & 0.087 & -.4191223 & .0311087 \\
\hline LD. & .054757 & .2268936 & 0.24 & 0.812 & -.4185348 & .5280488 \\
\hline _trend & .0197459 & .0096586 & 2.04 & 0.054 & -.0004015 & .0398934 \\
\hline _cons & 1.539988 & .8573523 & 1.80 & 0.088 & -.2484171 & 3.328394 \\
\hline
\end{tabular}

Fuente: Elaboración propia con base en DANE, 2016.

Anexo B. Prueba de raíz unitaria para el crecimiento del PIB.

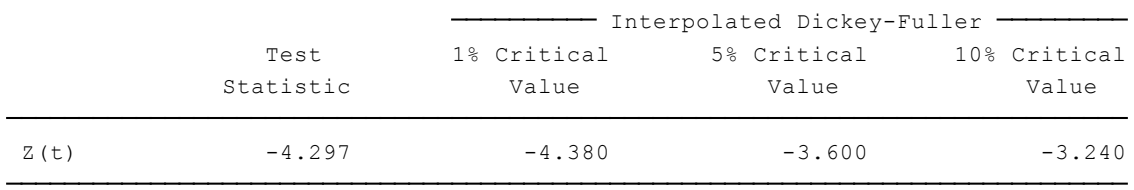

Mackinnon approximate $p$-value for $z(t)=0.0032$

\begin{tabular}{r|rrrrrr}
\hline D.Dlpibreal & Coef. & Std. Err. & $t$ & P $>|t|$ & [95\% Conf. Interval] \\
\hline Dlpibreal & & & & & & \\
L1. & -1.011745 & .2354524 & -4.30 & 0.000 & -1.501395 & -.5220951 \\
_trend & .0044087 & .0047623 & 0.93 & 0.365 & -.005495 & .0143125 \\
_cons & .0075728 & .0643559 & 0.12 & 0.907 & -.1262625 & .1414081 \\
\hline
\end{tabular}

Fuente: Elaboración propia con base en DANE, 2016.

Anexo C. Prueba de raiz unitaria para los precios.

\begin{tabular}{ccccc} 
& & \multicolumn{3}{c}{ Tnterpolated Dickey-Fuller } \\
\cline { 3 - 5 } & $\begin{array}{c}\text { To Critical } \\
\text { Stalustic }\end{array}$ & $\begin{array}{c}\text { 5\% Critical } \\
\text { Value }\end{array}$ & $\begin{array}{c}\text { 10\% Critical } \\
\text { Value }\end{array}$ \\
\hline$Z(t)$ & -3.573 & -4.380 & -3.600 & -3.240 \\
\hline
\end{tabular}

Mackinnon approximate $\mathrm{p}$-value for $\mathrm{z}(\mathrm{t})=0.0322$

\begin{tabular}{r|rrrrrr}
\hline D.IIPC_Vil v & Coef. & Std. Err. & $t$ & P > t I & [95\% Conf. Interval] \\
\hline lIPC_Villav & & & & & & \\
L1. & -.0815712 & .0228309 & -3.57 & 0.002 & -.1291956 & -.0339467 \\
LD. & .4607469 & .1678309 & 2.75 & 0.012 & .1106578 & .8108359 \\
-trend & .0029376 & .0019107 & 1.54 & 0.140 & -.001048 & .0069232 \\
_cons & .3409327 & .0907271 & 3.76 & 0.001 & .1516792 & .5301861 \\
\hline
\end{tabular}

Fuente: Elaboración propia con base en DANE, 2016. 
Anexo D. Prueba de raiz unitaria para la inflación.

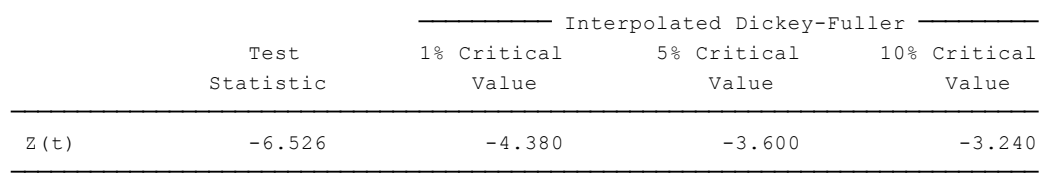

Mackinnon approximate $p$-value for $z(t)=0.0000$

\begin{tabular}{r|rrrrrr}
\hline D.dlIPC_Vi v & Coef. & Std. Err. & $t$ & P $>|t|$ & [95\% Conf. Interval] \\
\hline dlIPC_Villav & & & & & & \\
L1. & -1.347839 & .2065218 & -6.53 & 0.000 & -1.777325 & -.9183539 \\
_trend & -.0118718 & .0039999 & -2.97 & 0.007 & -.02019 & -.0035536 \\
_cons & .2763128 & .0670038 & 4.12 & 0.000 & .1369709 & .4156548 \\
\hline
\end{tabular}

Fuente: Elaboración propia con base en DANE, 2016.

Anexo E. Orden del VAR.

Selection-order criteria

Sample: $1997-2015$

\begin{tabular}{|c|cccccccc|}
\hline lag & LL & LR & df & p & FPE & AIC & HQIC & SBIC \\
\hline 0 & 28.7157 & & & & .000392 & -2.1806 & -2.1133 & -1.78294 \\
1 & 31.5214 & 5.6113 & 4 & 0.230 & .000459 & -2.05488 & -1.95393 & -1.45839 \\
2 & 43.1438 & $23.245 *$ & 4 & 0.000 & $.00022^{*}$ & $-2.85725^{*}$ & $-2.72265 *$ & $-2.06193^{*}$ \\
3 & 46.5783 & 6.869 & 4 & 0.143 & .000264 & -2.79772 & -2.62947 & -1.80357 \\
4 & 50.9262 & 8.6957 & 4 & 0.069 & .000316 & -2.83433 & -2.63243 & -1.64136 \\
5 & 52.0506 & 2.2488 & 4 & 0.690 & .000623 & -2.53164 & -2.29609 & -1.13983 \\
6 & 54.5588 & 5.0164 & 4 & 0.286 & .001495 & -2.37461 & -2.10541 & -.783976 \\
\hline
\end{tabular}

Endogenous: Dlpibreal dlIPC_Villav

Exogenous: dfinanciera dhipoteca dpetroleo_cons

Fuente: Elaboración propia con base en DANE, 2016.

Anexo F. Test de multiplicador de Lagrange.

Lagrange-multiplier test

\begin{tabular}{|r|rrr|}
\hline lag & chi2 & $d f$ & Prob > chi2 \\
\hline 1 & 1.7022 & 4 & 0.79032 \\
2 & 4.2493 & 4 & 0.37332 \\
3 & 5.6635 & 4 & 0.22573 \\
4 & 3.5732 & 4 & 0.46684 \\
5 & 1.4083 & 4 & 0.84274 \\
6 & 1.7515 & 4 & 0.78134 \\
7 & 2.5730 & 4 & 0.63162 \\
8 & 0.1654 & 4 & 0.99676 \\
9 & 1.4318 & 4 & 0.83864 \\
10 & 7.6845 & 4 & 0.10384 \\
11 & 2.6158 & 4 & 0.62403 \\
12 & 3.8458 & 4 & 0.42728 \\
13 & 5.7985 & 4 & 0.21471 \\
14 & 5.5934 & 4 & 0.23164 \\
15 & 8.8020 & 4 & 0.06624 \\
16 & 1.6958 & 4 & 0.79148 \\
\hline
\end{tabular}

HO: no autocorrelation at lag order

Fuente: elaboración propia con base en DANE, 2016. 
Anexo G. Test de Portmanteau para ruido blanco.

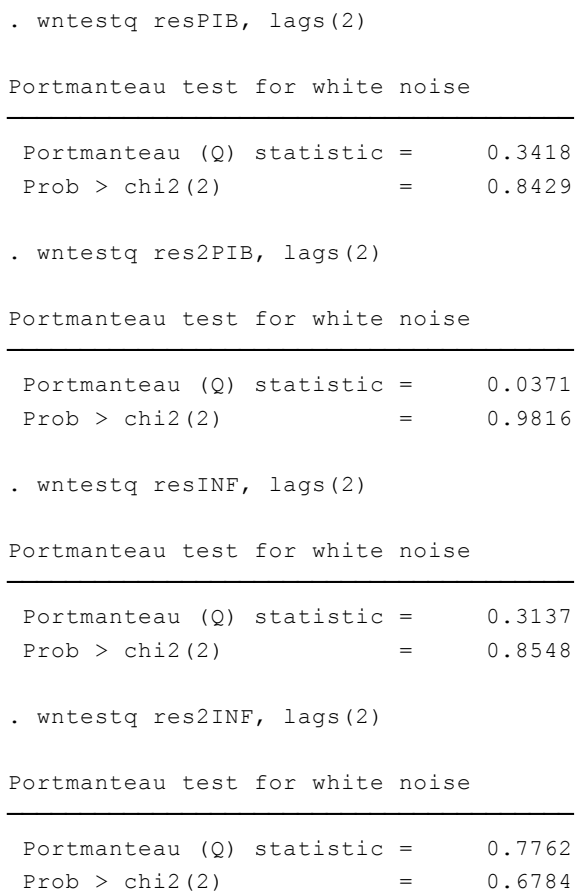

Fuente: Elaboración propia con base en DANE, 2016.

\section{Anexo H. Condición de estabilidad de los valores propios.}

Eigenvalue stability condition

\begin{tabular}{|c|c|}
\hline Eigenvalue & Modulus \\
\hline .9186759 & .918676 \\
-.6697397 & .66974 \\
.5434799 & .54348 \\
-.3779258 & .377926 \\
\hline
\end{tabular}

All the eigenvalues lie inside the unit circle. VAR satisfies stability condition.

Fuente: Elaboración propia con base en DANE, 2016. 
Astrid León-Camargo es Economista. Magíster en Ciencias Económicas. Magíster en Integración económica. Especialista en Finanzas, preparación y evaluación de proyectos. Especialista en Didácticas de lectura y escritura. Docente de la Universidad de los Llanos. Grupo de investigación "Integración", Facultad de Ciencias Económicas, Universidad de los Llanos. 